\title{
Analogies between Logistic Equation and Relativistic Cosmology
}

\author{
Steve Dussault ${ }^{1}$, Valerio Faraoni ${ }^{1, *}$ (i) and Andrea Giusti ${ }^{2}$ \\ 1 Department of Physics \& Astronomy, Bishop's University, Sherbrooke, QC J1M 1Z7, Canada; \\ sdussault19@ubishops.ca \\ 2 Institute for Theoretical Physics, ETH Zurich, Wolfgang-Pauli-Strasse 27, 8093 Zurich, Switzerland; \\ agiusti@phys.ethz.ch \\ * Correspondence: vfaraoni@ubishops.ca; Tel.: +1-819-822-9600 (ext. 2490); Fax: +1-819-822-9611
}

Citation: Dussault, S.; Faraoni, V.; Giusti, A. Analogies between Logistic Equation and Relativistic Cosmology. Symmetry 2021, 13, 704. https:// doi.org/10.3390/sym13040704

Received: 26 March 2021

Accepted: 15 April 2021

Published: 17 April 2021

Publisher's Note: MDPI stays neutral with regard to jurisdictional claims in published maps and institutional affiliations.

\begin{abstract}
We develop several formal analogies between the logistic equation and the spatially homogeneous and isotropic relativistic cosmology described by the Einstein-Friedmann equations. These analogies produce an effective Lagrangian and Hamiltonian and new symmetries for the logistic equation.
\end{abstract}

Keywords: logistic equation; relativistic cosmology; analogies

\section{Introduction}

The logistic equation is

$$
\dot{f}(t)=r f(t)[1-f(t)],
$$

where $r$ is a positive constant and an overdot denotes differentiation with respect to $t$. The solution is the well known sigmoid

$$
f(t)=\frac{f_{0} \mathrm{e}^{r t}}{1+f_{0} \mathrm{e}^{r t}}
$$

where $f_{0}$ is a constant. If $f_{0}<-1$, the initial value at $t=0$ is $f(0)=\frac{f_{0}}{1+f_{0}}=\frac{\left|f_{0}\right|}{\left|f_{0}\right|-1}>1$ and $\dot{f}<0$. This solution decreases and approaches 1 asymptotically. If instead $f_{0}>0$, then $f(0)<1$ and the solution always satisfies $f<1$. We require $f \geq 0$ and we restrict to the range $0<f \leq 1$.

Other solutions are the constants $f \equiv 1$, which is a fixed point solution and a late-time attractor, and $f(t) \equiv 0$ which is also a fixed point and a repellor but is not considered here since we restrict to positive $f$.

The logistic equation and its applications are well known: here, we point out a novel aspect of it, i.e., formal analogies between the logistic equation and the Friedmann equation of spatially homogenous and isotropic cosmology in general relativity. There are two analogs, corresponding to the two different time coordinates widely used in cosmology. First, these analogies generate an effective Lagrangian and Hamiltonian for the logistic equation or, in other words, solve its inverse Lagrangian problem. Second, the Friedmann equation admits certain symmetries which can be used to generate corresponding symmetries on the logistic equation side of the analogy.

In the next section, we recall the basics of spatially homogeneous and isotropic cosmology (also called Friedmann-Lemaître-Robertson-Walker (FLRW) cosmology) in Einstein's theory of gravity and the symmetries of the corresponding Einstein-Friedmann equations. In the following section, we develop the formal analogies between logistic equation and Friedmann equation, written using comoving time or conformal time, respectively. In both cases, we write down the corresponding Lagrangian and Hamiltonian for the logistic equation, and examine whether the symmetries of the Einstein-Friedmann equations generate new symmetries of the logistic equation. The last section contains the conclusions. 


\section{Basics of FLRW Cosmology}

The foundation of relativistic cosmology is the Copernican principle, according to which the universe is spatially homogeneous and isotropic on large scales (i.e., averaging over spatial regions of size larger than approximately 100 Megaparsecs) [1-6]. Therefore, the relativistic spacetime manifold used to model the universe is taken to satisfy the symmetry requirements of spatial homogeneity and isotropy. The latter are quite stringent: in fact, the spacetime manifold must have constant spatial curvature [7]. The spacetime geometry must then necessarily be the one described by the FLRW metric tensor. Using polar comoving coordinates (the comoving coordinates are associated with observers comoving with the cosmic fluid, i.e., those who see the cosmic microwave background radiation homogeneous and isotropic around them (apart from tiny temperature perturbations of the order $\left.\left.\delta T / T \sim 5 \times 10^{-5}\right)\right)(t, r, \vartheta, \varphi)$, the FLRW line element is [1,2]

$$
d s^{2}=-d t^{2}+a^{2}(t)\left(\frac{d r^{2}}{1-K r^{2}}+r^{2} d \Omega_{(2)}^{2}\right)
$$

where $d \Omega_{(2)}^{2}=d \vartheta^{2}+\sin ^{2} \vartheta d \varphi^{2}$ denotes the line element on the unit 2-sphere, while the sign of the constant "curvature index" $K$ characterizes the three-dimensional spatial sections corresponding to instants of comoving time $t=$ const. A positive curvature index $K>0$ is associated with 3-spheres; vanishing curvature $K=0$ corresponds to Euclidean spatial sections, while an index $K<0$ is associated with hyperbolic three-dimensional spatial sections.

\subsection{Einstein-Friedmann Equations}

Thanks to the symmetries, the entire evolution of FLRW universes is described by the functional form of the scale factor $a(t)$. The spacetime geometry is curved by some matter source: in FLRW cosmology, it is customary (but not compulsory) to use as the matter source a perfect fluid with energy density $\rho(t)$ and pressure given by $P(t)=w \rho(t)$. Here, $w$ denotes a constant equation of state parameter. The evolution of the cosmic scale factor $a(t)$ and energy density $\rho(t)$ is ruled by the Einstein equations, which are greatly simplified by the high degree of symmetry of FLRW cosmology and reduce to a set of three ordinary differential equations, the Einstein-Friedmann equations. These equations include [1-3] the Friedmann equation

$$
H^{2}=\frac{8 \pi G}{3} \rho-\frac{K}{a^{2}}+\frac{\Lambda}{3}
$$

(which amounts to a first order constraint on the dynamics); the acceleration (or Raychaudhuri) equation

$$
\frac{\ddot{a}}{a}=-\frac{4 \pi G}{3}(\rho+3 P)+\frac{\Lambda}{3} ;
$$

and the equation describing covariant conservation of energy of the cosmic fluid

$$
\dot{\rho}+3 H(P+\rho)=0 .
$$

The constants appearing in these equations are Newton's constant $G$ and Einstein's celebrated cosmological constant $\Lambda$. We use an overdot to indicate differentiation with respect to the comoving time $t$, while $H \equiv \dot{a} / a$ is the Hubble function [2,3]. The cosmological constant $\Lambda$ can be treated formally as an effective perfect fluid with energy density and pressure $\rho_{\Lambda}=\frac{\Lambda}{8 \pi G}=-P_{\Lambda}[2,3]$. Following the notation in [2,3], we use units in which the speed of light is unity.

For a generic cosmic fluid, only two out of the three Equations (4)-(6) are independent and one of them can be derived from the other two, if the Einstein equations and the expression of the Ricci scalar in FLRW space are used as extra information. Specifically: 
- Differentiating the Friedmann Equation (4) with respect to time yields

$$
2 H\left(\frac{\ddot{a}}{a}-H^{2}\right)=\frac{8 \pi G}{3} \dot{\rho}+\frac{2 K H}{a^{2}} ;
$$

using the acceleration Equation (5) and the Friedmann Equation (4) to substitute for $\ddot{a} / a$ and $H^{2}$ in (7) produces the conservation Equation (6). This derivation of Equation (6) is a reflection of the more general derivation of the covariant conservation equation $\nabla^{b} T_{a b}=0$ from the Einstein equations

$$
R_{a b}-\frac{1}{2} g_{a b} R+\Lambda g_{a b}=8 \pi G T_{a b}
$$

and the contracted Bianchi identities $\nabla^{b}\left(R_{a b}-g_{a b} R / 2\right)=0$ (here, $g_{a b}, R_{a b}$, and $T_{a b}$ are the metric tensor, its Ricci tensor, and the stress-energy tensor of matter, respectively, and $\left.R \equiv g^{a b} R_{a b}\right)$.

- The Friedmann Equation (4) follows from the acceleration Equation (5), the Einstein Equation (8), and the expression of the Ricci scalar in the FLRW geometry (3)

$$
R=6\left(\dot{H}+2 H^{2}+\frac{K}{a^{2}}\right) .
$$

In fact, a perfect fluid stress-energy tensor

$$
T_{a b}=(P+\rho) u_{a} u_{b}+P g_{a b}
$$

(where $u^{c}$ is the fluid four-velocity normalized to $u^{c} u_{c}=-1$ ) has trace $T=-\rho+3 P$ and the contraction of the Einstein Equations (8) then gives

$$
R=8 \pi G(\rho-3 P)+4 \Lambda .
$$

Equating the right-hand sides of Equations (9) and (11) yields

$$
\frac{\ddot{a}}{a}+H^{2}+\frac{K}{a^{2}}=\frac{4 \pi G}{3}(\rho-3 P)+\frac{2 \Lambda}{3} .
$$

Using now the acceleration Equation (5) to substitute for $\ddot{a} / a$, one obtains the Friedmann Equation (4).

- The acceleration Equation (5) can be derived from the Friedmann Equation (4) and the energy conservation Equation (6). In fact, differentiating (4) with respect to time leads to

$$
2 H\left(\frac{\ddot{a}}{a}-H^{2}\right)=\frac{8 \pi G}{3} \dot{\rho}+\frac{2 K H}{a^{2}} ;
$$

using Equation (6) to substitute for $\dot{\rho}$ and the Friedmann Equation (4) for $H^{2}$, the acceleration Equation (5) follows straightforwardly.

By specifying a constant equation of state $P=w \rho$, the conservation equation is immediately integrated to

$$
\rho(a)=\frac{\rho_{0}}{a^{3(w+1)}} .
$$

Analytic solutions of the Einstein-Friedmann Equations (4)-(6) with a single perfect fluid, as well as analyses of their phase space, are well known (see [8-10] for reviews).

\subsection{FLRW Lagrangian and Hamiltonian}

A Lagrangian for FLRW cosmology is

$$
L(a, \dot{a})=3 a \dot{a}^{2}+8 \pi G a^{3} \rho-3 K a,
$$


where $\rho=\rho(a)$ is specified by the barotropic equation of state $P=P(\rho)$ and the conservation equation $\dot{\rho}+3 H(P+\rho)=0$. Since $\rho=\rho(a)$, the Lagrangian (15) does not depend explicitly on $t$ and the corresponding Hamiltonian is conserved,

$$
\mathcal{H}=\frac{\partial L}{\partial \dot{a}} \dot{a}-L=3 a \dot{a}^{2}-8 \pi G a^{3} \rho+3 K a=C .
$$

The Lagrangian and Hamiltonian above solve the inverse variational problem of finding an action integral for the logistic equation, however this is not sufficient to complete the derivation. Since the dynamics of general relativity is constrained [2,3], in the FLRW geometry the Hamiltonian constraint (time-time component of the Einstein equations) imposes that $C=0$ and is the same as the Friedmann Equation (4) [2].

\subsection{Symmetries of the Einstein-Friedmann Equations for Spatially Flat Universes}

If the universe is spatially flat $(K=0)$ and there is a single term on the right-hand side of the Friedmann Equation (4), corresponding to a single perfect fluid with constant equation of state $P=w \rho$, the Einstein-Friedmann equations exhibit certain symmetries [11-13] which are studied in the cosmological literature for the purpose of generating analytic solutions [11-32]. In these symmetry operations, time $t$, scale factor $a$, or Hubble function $H$ are rescaled and the cosmic fluid is changed appropriately, leaving the Einstein-Friedmann equations invariant in form.

Under the first symmetry [12]

$$
\begin{aligned}
a \rightarrow \tilde{a} & =\frac{1}{a}, \\
w \rightarrow \tilde{w} & =-(w+2),
\end{aligned}
$$

an expanding universe changes into a contracting one and vice versa. (When it is applicable to the logistic equation, this symmetry will change an increasing solution with $f<1$ into a decreasing one with $f>1$.)

Another one-parameter group of symmetry transformations of a $K=0$ universe is [13]

$$
\begin{aligned}
a & \rightarrow \bar{a}=a^{s}, \\
d t & \rightarrow d \bar{t}=s a^{\frac{3(w+1)(s-1)}{2}} d t, \\
\rho & \rightarrow \bar{\rho}=a^{-3(w+1)(s-1)} \rho,
\end{aligned}
$$

where the real number $s \neq 0$ parameterizes the transformation. These operations form a one-parameter Abelian group.

Finally, the third type of symmetry for a spatially flat FLRW cosmology is $[11,33]$

$$
\begin{aligned}
& \rho \rightarrow \bar{\rho}=\bar{\rho}(\rho), \\
& H \rightarrow \bar{H}=\sqrt{\frac{\bar{\rho}}{\rho}} H, \\
& P \quad \rightarrow \quad \bar{P}=-\bar{\rho}+\sqrt{\frac{\rho}{\bar{\rho}}}(P+\rho) \frac{d \bar{\rho}}{d \rho},
\end{aligned}
$$

where the function $\bar{\rho}(\rho)$ has the same sign as $\rho$ and is regular. This symmetry applies also when the barotropic perfect fluid in the FLRW universe does not have linear or constant equation of state. 


\section{Cosmological Analogies}

Dividing the logistic Equation (1) by $f$ and squaring gives

$$
\left(\frac{\dot{f}}{f}\right)^{2}=r^{2}-2 r^{2} f+r^{2} f^{2} .
$$

In the following, we examine analogies with the Friedmann Equation (4).

\subsection{First Analogy Using Comoving Time}

Equation (25) is formally analogous to the Friedmann Equation (4) with $(t, a(t)) \leftrightarrow$ $(t, f(t)), K=0, \Lambda=3 r^{2}$, and energy density

$$
\rho=\rho_{0} a(a-2)
$$

with $\rho_{0}=3 r^{2} /(8 \pi G)$. (In FLRW cosmology, the scale factor can be rescaled by a constant without affecting the physics. However, the solution $f(t)$ of the logistic equation does not enjoy this property, because of the quantity $1-f$ appearing in brackets in the right hand side of Equation (1). By imposing the analogy between $a(t)$ and $f(t)$, one loses this property of the scale factor $a(t)$.) If the physical requirement that the energy density be nonnegative is imposed, the scale factor must satisfy the lower bound $a(t) \geq 2 \forall t$. However, one can in principle relax this assumption if one is only interested in the mathematical properties of this formal analogy. To complete the analogy, one must impose that another of the Einstein-Friedmann equations be satisfied. For simplicity, we choose to impose the covariant conservation equation $\dot{\rho}+3 H(P+\rho)=0$, which then yields the effective equation of state of the cosmic fluid

$$
P=-\rho-\frac{a}{3} \frac{d \rho}{d a} .
$$

Equation (26) can be sen as the quadratic $a^{2}-2 a-\rho / \rho_{0}=0$; for physical reasons only the positive root

$$
a=1+\sqrt{1+\frac{\rho}{\rho_{0}}}
$$

is relevant in cosmology, hence, we choose the positive sign and restrict ourselves to $f>0$ in the logistic equation. Substituting Equation (28) into Equation (27) yields the nonlinear equation of state of the analogous cosmic fluid

$$
P=-\frac{5}{3} \rho-\frac{2 \rho_{0}}{3}\left[1+\sqrt{1+\frac{\rho}{\rho_{0}}}\right],
$$

which describes a phantom fluid (i.e., $P / \rho<-1$ ). To summarize, this analogous universe is spatially flat, has positive cosmological constant, and is filled with an exotic phantom perfect fluid. From a physical point of view, the energy density must be non-negative, which implies $a \geq 2$, while the sigmoid (2) is never larger than one. Therefore, $\rho<0$ and one should discard this first analogy from the physical point of view. However, if only the mathematical properties are of interest, the formal analogy still stands.

Lagrangian and Hamiltonian. One can consider the inverse variational problem for Equation (1). The cosmological analogy gives the Lagrangian for the logistic equation

$$
L_{1}(f, \dot{f})=f \dot{f}^{2}+r^{2} f^{4}(f-2)+r^{2} f^{3} .
$$

Since $L_{1}$ does not depend explicitly on time, the corresponding Hamiltonian is conserved,

$$
\mathcal{H}_{1}=\frac{\partial L_{1}}{\partial \dot{f}} \dot{f}-L_{1}=f \dot{f}^{2}-r^{2} f^{4}(f-2)-r^{2} f^{3}=C_{1} .
$$


Setting the constant $C_{1}=0$ yields $\dot{f}= \pm|r| f(1-f)$. The negative sign corresponds to inverting the sign of the constant $r$, which was stipulated to be positive from the beginning. Symmetries. Since the equation of state is non-linear, the symmetries (17) and (18) and (19)-(21) do not apply to this analogous FLRW universe.

The third symmetry is enjoyed by an equation of the form (4) with a single perfect fluid or with a single fluid plus cosmological constant [33]. In the present situation, we necessarily have two fluids plus cosmological constant because eliminating one of the fluids necessarily implies setting $r=0$, which leads to losing the logistic equation. As a conclusion, the third symmetry does not apply to Equation (25).

\subsection{Second Analogy with Comoving Time}

As a second possible analogy, one can take instead a spatially flat $(K=0)$ universe with $\Lambda=0$ and energy density

$$
\rho=\rho_{0}(1-a)^{2}
$$

imposing the covariant energy conservation equation yields

$$
P=-\rho+\frac{2 \rho_{0}}{3} a(1-a)
$$

and, eliminating $a$ with Equation (32), which yields $a=1 \pm \sqrt{\rho / \rho_{0}}$, one obtains the nonlinear equation of state

$$
P=-\frac{5}{3} \rho \mp \frac{2}{3} \sqrt{\rho_{0} \rho} .
$$

This time, the energy density is automatically non-negative and this is an acceptable analogy from the physical point of view. The FLRW universe analogous to the logistic equation is spatially flat, has zero cosmological constant, and is filled with the exotic fluid (34). Since $P<-\rho / 3$, this universe is accelerated, as follows from the acceleration equation $\frac{\ddot{a}}{a}=-\frac{4 \pi G}{3}(\rho+3 P)$.

Lagrangian and Hamiltonian. The Lagrangian and Hamiltonian for this analogous universe

$$
\begin{aligned}
L_{2} & =3 a \dot{a}^{2}+8 \pi G \rho_{0} a^{3}(1-a)^{2}, \\
\mathcal{H}_{2} & =3 a \dot{a}^{2}-8 \pi G \rho_{0} a^{3}(1-a)^{2},
\end{aligned}
$$

reproduce (apart from an irrelevant multiplicative constant) the Lagrangian (30) and Hamiltonian (31) for the logistic equation.

Symmetries. Since the equation of state is non-linear, the symmetries (17) and (18) and (19)-(21) do not apply to this analogous FLRW universe. The remaining FLRW symmetry (22)-(24) preserves the analogy (and, therefore, the logistic equation) provided that the equation of state (34) is maintained. This requirement implies that

$$
\pm \bar{\rho}^{3 / 2}+\sqrt{\bar{\rho}_{0}} \bar{\rho}=\left( \pm \rho^{3 / 2}+\sqrt{\rho_{0}} \rho\right) \frac{d \bar{\rho}}{d \rho}
$$

and, consequently,

$$
\int \frac{d \bar{\rho}}{\sqrt{\bar{\rho}_{0}} \bar{\rho} \pm \bar{\rho}^{3 / 2}}=\int \frac{d \rho}{\sqrt{\rho_{0}} \rho \pm \rho^{3 / 2}} .
$$

Computing the integral, one obtains

$$
\frac{2}{\sqrt{\overline{\rho_{0}}}} \ln \left(\frac{\sqrt{\bar{\rho}}}{\sqrt{\bar{\rho}} \pm \sqrt{\overline{\rho_{0}}}}\right)=\frac{2}{\sqrt{\rho_{0}}} \ln \left(\frac{\sqrt{\rho}}{\sqrt{\rho} \pm \sqrt{\rho_{0}}}\right)
$$


which is conveniently rewritten as

$$
\ln \left(1 \pm \sqrt{\frac{\bar{\rho}_{0}}{\bar{\rho}}}\right)=\sqrt{\frac{\bar{\rho}_{0}}{\rho_{0}}} \ln \left(1 \pm \sqrt{\frac{\rho_{0}}{\rho}}\right) .
$$

Using the fact that

$$
\sqrt{\frac{\bar{\rho}_{0}}{\rho_{0}}}=\frac{\bar{r}}{r}
$$

one obtains

$$
1 \pm \sqrt{\frac{\bar{\rho}_{0}}{\bar{\rho}}}=\left(1 \pm \sqrt{\frac{\rho_{0}}{\rho}}\right)^{\bar{r} / r}
$$

so that

$$
1 \pm \sqrt{\frac{\rho_{0}}{\rho}}=\frac{1 \pm 1-f}{1-f} ;
$$

let us adopt the lower sign first. Then, Equation (42) yields

$$
-\frac{\bar{f}}{1-\bar{f}}=\left(-\frac{f}{1-f}\right)^{\bar{r} / r}
$$

which, since the argument of the parenthesis in the right hand side is negative, is well defined only if $\bar{r} / r \equiv n$ is an integer. If $n$ is even, Equation (44) has no solutions because the left hand side is negative while the right hand side is positive. If instead $n=2 k+1$, $k \in \mathbb{N}$, then (44) reduces to

$$
f \rightarrow \bar{f}=\frac{\left(\frac{f}{1-f}\right)^{2 k+1}}{1+\left(\frac{f}{1-f}\right)^{2 k+1}} .
$$

The sigmoid solution (2) of the logistic equation clearly enjoys this symmetry, which corresponds to the simple rescaling

$$
f=\frac{f_{0} \mathrm{e}^{\beta t}}{1+f_{0} \mathrm{e}^{\beta t}} \rightarrow \bar{f}=\frac{\bar{f}_{0} \mathrm{e}^{\bar{\beta} t}}{1+\bar{f}_{0} \mathrm{e}^{\bar{\beta} t}}
$$

with

$$
\begin{aligned}
\bar{f}_{0} & =f_{0}^{2 k+1}, \\
\bar{\beta} & =(2 k+1) \beta,
\end{aligned}
$$

which, alternatively, follows directly from the properties of the exponential function in the solution.

If we instead adopt the upper sign in Equation (43), we obtain

$$
\begin{aligned}
\frac{2-\bar{f}}{1-\bar{f}} & =\left(\frac{2-f}{1-f}\right)^{\bar{r} / r} \equiv \gamma, \\
\bar{f} & =\frac{2-\gamma}{1-\gamma},
\end{aligned}
$$

from which it follows that the solution (2) is mapped into

$$
\bar{f}=1+\frac{1}{1-\left(2+f_{0} \mathrm{e}^{\beta t}\right)^{\bar{r} / r}} .
$$


Certain symmetries of the logistic equation are hidden and made manifest by the analogy with cosmology. Otherwise, it is not obvious how to look for such symmetries, either in the equation or in its solutions. This is the case, for example, of the symmetries described by Equation (45) or Equation (51), which apparently are not found in systematic searches for symmetries.

\subsection{First Analogy with Conformal Time}

One can rewrite the Friedmann Equation (4) using conformal, instead of comoving, time. The conformal time $\eta$ is defined by $d t \equiv a d \eta$ [2]. Assume that there is a single perfect fluid with constant equation of state $P=w \rho$ and $\Lambda=0$. Then, the use of conformal time turns the combined Friedmann Equation (4) and acceleration equation $\frac{\ddot{a}}{a}=-\frac{4 \pi G}{3}(3 w+1) \rho$ into

$$
\frac{a_{\eta \eta}}{a}+(c-1)\left(\frac{a_{\eta}}{a}\right)^{2}+c K=0,
$$

where $c=(3 w+1) / 2$. Using the new variable $u \equiv a_{\eta} / a$, Equation (52) is reduced to the Riccati equation [9]

$$
u_{\eta}+c u^{2}+c K=0 .
$$

This reduction is a known way of solving the Einstein-Friedmann equation for a universe filled with a perfect fluid with constant equation of state, which is an alternative to the more standard solution method using quadratures [9,34]. It has been used as the basis for several applications in cosmology [35-44].

The logistic equation can also be reduced to a Riccati equation [43]. From a broader point of view, this is not the only integrable first-order equation that connects with FLRW universes, especially those filled with scalar fields. For example, the solution of the Riccati Equation (53) is a hyperbolic tangent, while cosmological models based on a phantom scalar field expressed by an hyperbolic tangent are known [45]. Another first-order equation used to integrate a cosmological model based on a scalar field with exponential potential appears in [46]. Another connection between scalar field cosmology and the Abel equation of the first kind was investigated by Yurov and Yurov [47]. More generally, connections between the Einstein-Friedmann equations and the Ermakov-Pinney equation [48] and the Schrödinger equation are known $[49,50]$.

Proceeding with the Riccati Equation (53) and setting

$$
f(t) \equiv \frac{1}{2}[g(t)+1]
$$

Equation (1) becomes

$$
\dot{g}+\frac{r}{2} g^{2}-\frac{r}{2}=0,
$$

which is of the form (53) with $c=r / 2, K=-1$. We have then the formal analogy $(\eta, u(\eta)) \rightarrow(t, g(t))=(t, 2 f(t)-1)$ provided that $K=-1$ and $c=r / 2=(3 w+1) / 2$, which gives $w=(r-1) / 3$. Two special cases correspond to classic solutions of FLRW cosmology [2-4,6]: $r=1$ corresponds to a dust (zero pressure fluid), while $r=2$ corresponds to a radiation fluid, both of which are solved exactly [2].

The analogy implies that

$$
\frac{a_{\eta}}{a}=u=g(\eta)=2 f(\eta)-1 ;
$$

using the solution (2) and integrating gives the scale factor of the analogous universe in conformal time

$$
a(\eta)=a_{0} \mathrm{e}^{-\eta}\left(1+f_{0} \mathrm{e}^{r \eta}\right)^{2 / r} .
$$


The comoving time $t$ is obtained from the conformal time by integration, producing the hypergeometric function

$$
t=\int a d \eta=-a_{0} \mathrm{e}^{-\eta}{ }_{2} F_{1}\left(-\frac{2}{r},-\frac{1}{r}, \frac{r-1}{r},-f_{0} \mathrm{e}^{r \eta}\right)+t_{0},
$$

where $t_{0}$ is an integration constant. Equations (57) and (58) constitute a parametric representation of the solution $a(t)$ in terms of comoving time with $\eta$ as the parameter. Unfortunately, this representation is too cumbersome for practical uses in most situations, and it is more convenient to use $a(\eta)$ instead.

Lagrangian and Hamiltonian. A Lagrangian for the Riccati Equation (55) is (the Lagrangian formalism for second-order Riccati equations different from the present one was studied by Cari nena, Ra nada, and Santander [44])

$$
L_{R}(g, \dot{g})=\dot{g}^{2}+\frac{r^{2}}{4}\left(g^{2}-1\right)^{2},
$$

which does not depend explicitly on $\eta$, hence the corresponding Hamiltonian

$$
\mathcal{H}_{R}=\dot{g}^{2}-\frac{r^{2}}{4}\left(g^{2}-1\right)^{2}
$$

is conserved. Choosing zero value for this constant and the negative sign of the root of $\mathcal{H}=0$ reproduces Equation (55).

Symmetries. A priori, the symmetries (17) and (18) and (19)-(21) should not apply to the equation $u_{\eta}+c u^{2}-c=0$ because this implies $K=-1$, However, the first symmetry translates into

$$
u \rightarrow \bar{u}=\frac{1}{u}
$$

which leaves this equation invariant. As expected, the second FLRW symmetry does not translate into useful symmetries of the Riccati equation. The third symmetry, valid for spatially flat FLRW universes, does not apply here because $K$ is fixed to the value -1 .

\subsection{Second Analogy with Conformal Time}

Instead of the analogy $(\eta, u(\eta)) \rightarrow(t, f(t))$, let us consider now the more direct analogy $(\eta, a(\eta)) \rightarrow(t, f(t))$. The Friedmann equation in conformal time reads

$$
\left(\frac{a_{\eta}}{a}\right)^{2}=\frac{\Lambda a^{2}}{3}+\frac{8 \pi G}{3} \rho a^{2}-K
$$

which is analogous to Equation (25) if we make the correspondence

$$
\begin{aligned}
K & =-r^{2}, \\
\Lambda & =3 r^{2} \\
\rho & =-\frac{3 r^{2}}{4 \pi G} \frac{1}{a} \equiv-\frac{\rho_{0}}{a} .
\end{aligned}
$$

The energy density (65) is negative, which would lead to rejecting this analogy on purely physical grounds.

Lagrangian and Hamiltonian. The usual Lagrangian can be used, provided that the cosmological constant is treated as an extra perfect fluid with energy density $\rho_{\Lambda}=\Lambda /(8 \pi G)$ added to the usual perfect fluid, which yields

$$
L_{3}=\frac{a_{\eta}^{2}}{a}+\frac{8 \pi G}{3} \rho a^{3}-K a+\frac{\Lambda a^{2}}{3}
$$


and the Hamiltonian

$$
\mathcal{H}_{3}=\frac{a_{\eta}^{2}}{a}-\frac{8 \pi G}{3} \rho a^{3}+K a-\frac{\Lambda a^{2}}{3} .
$$

Using the identifications (63)-(65), the logistic equation is equivalent to $\mathcal{H}_{3}=0$ (with the choice of the positive sign when taking the square root of both sides).

Symmetries. Since the spatial sections of this universe are spatially curved, the symmetries (17) and (18) and (19)-(21) do not apply to this analogous FLRW universe. The third symmetry is enjoyed by an equation of the form (4) with a single perfect fluid or with a single fluid plus cosmological constant [33]. In our case, eliminating the second fluid (or $K$-fluid) by setting $K=0$ means setting $r=0$ and losing the logistic equation altogether. Therefore, the third symmetry does not apply to Equation (62).

\section{Discussion and Conclusions}

On the basis of the previous analogies, we can extend the knowledge of solutions of the Einstein-Friedmann equations to those of the logistic equation. In particular, certain recent results on the analytic solutions of the Einstein-Friedmann equations [51-53] can be immediately transposed to the logistic equation. First, the work of Chen, Gibbons, and Yang [53] contains an explicit proof that all solutions of the Friedmann Equation (4) are roulettes. Therefore, one concludes without effort that all solutions of the logistic equation are also roulettes. [A roulette is the trajectory in two dimensions described by a point that lies on a curve rolling without slipping on another given curve.]

Second, one wonders under which conditions it is possible to obtain analytic solutions of the Einstein-Friedmann equations in terms of elementary functions. This non-trivial question was answered by Chen,Gibbons, Li, and Yang [51] using Chebysev's theorem of integration $[54,55]$. The transposition of this result to the logistic equation does not provide new information, since the solution of the latter is the well known sigmoid.

Third, one can deduce effective Lagrangians and Hamiltonians for the logistic equation (and also for a special Riccati equation appearing in the cosmological analogy using conformal time). The most important consequence of the Lagrangian formulation is probably that these systems admit a conserved energy function. Parallel to the fact that the dynamics of FLRW (and, in a wider context, of general relativity) is constrained, this conserved energy is forced to have zero value. Furthermore, these Lagrangian and Hamiltonian are obtained by squaring a first-order differential equation, thus introducing one extra mode with respect to the original equation. Hence, a sign choice must be made in order to reproduce the correct equation that we started from (see [56] for a more comprehensive discussion of this procedure). Certain symmetries of the EinsteinFriedmann equations unveil hidden symmetries of the logistic equation. It is currently not clear whether these symmetries are useful for applications of the logistic equation.

It seems that the cosmology side of the analogy has less to gain. However, viewing the same problem from a different angle is often interesting a priori. For example, it is interesting to see the evolution of the scale factor $a(t)$ for the phantom fluid examined here on par with the evolution of a population described by Verhulst with the logistic equation.

Author Contributions: Conceptualization, S.D. and A.G.; Formal analysis, S.D., V.F. and A.G.; Funding acquisition, V.F.; Investigation, S.D., V.F. and A.G.; Methodology, S.D., V.F. and A.G.; Supervision, V.F.; Writing-original draft, V.F.; Writing—review and editing, A.G. All authors have read and agreed to the published version of the manuscript.

Funding: This work was supported, in part, by the Natural Sciences \& Engineering Research Council of Canada (Grant No. 2016-03803 to V. Faraoni) and by Bishop's University. A. Giusti is supported by the European Union's Horizon 2020 research and innovation programme under the Marie SkłodowskaCurie Actions (grant agreement No. 895648-CosmoDEC). His work has been carried out in the framework of the activities of the Italian National Group for Mathematical Physics [Gruppo Nazionale per la Fisica Matematica (GNFM), Istituto Nazionale di Alta Matematica (INdAM)].

Institutional Review Board Statement: Not applicable. 
Informed Consent Statement: Not applicable.

Acknowledgments: We thank the two referees for constructive comments.

Conflicts of Interest: The authors declare no conflict of interest

\section{References}

1. Hawking, S.W.; Ellis, G.F.R. The Large Scale Structure of Space-Time; Cambridge University Press: Cambridge, UK, 1973.

2. Wald, R.M. General Relativity; Chicago University Press: Chicago, IL, USA, 1984.

3. Carroll, S.M. Spacetime and Geometry: An Introduction to General Relativity; Addison Wesley: San Francisco, CA, USA, 2004.

4. Kolb, E.W.; Turner, M.S. The Early Universe; Addison-Wesley: Redwood City, CA, USA, 1990.

5. Liddle, A.R.; Lyth, D.H. Cosmological Inflation and Large-Scale Structure; Cambridge University Press: Cambridge, UK, 2000.

6. $\quad$ Liddle, A.R. An Introduction to Modern Cosmology; Wiley: New York, NY, USA, 2015.

7. Eisenhart, L.P. Riemannian Geometry; Princeton University Press: Princeton, NJ, USA, 1949.

8. Felten, J.E.; Isaacman, R. Scale factors $R(t)$ and critical values of the cosmological constant $\Lambda$ in Friedmann universes. Rev. Mod. Phys. 1986, 58, 689. [CrossRef]

9. Faraoni, V. Solving for the dynamics of the universe. Am. J. Phys. 1999, 67, 732. [CrossRef]

10. Sonego, S.; Talamini, V. Qualitative study of perfect-fluid Friedmann-Lemaître-Robertson-Walker models with a cosmological constant. Am. J. Phys. 2012, 80, 670. [CrossRef]

11. Chimento, L.P. Symmetry and inflation. Phys. Rev. D 2002, 65, 063517. [CrossRef]

12. Faraoni, V. A symmetry of the spatially flat Friedmann equations with barotropic fluids. Phys. Lett. B 2011, 703, 228. [CrossRef]

13. Faraoni, V. A Symmetry of the Einstein-Friedmann Equations for Spatially Flat, Perfect Fluid, Universes. Symmetry 2020, $12,147$. [CrossRef]

14. Szydlowski, M.; Heller, M. Equation of state and equation symmetries in cosmology. Acta Phys. Polon. 1983, B14, 571.

15. Szydlowski, M.; Godlowski, W.; Wojtak, R. Equation of state for the Universe from similarity symmetries. Gen. Relativ. Gravit. 2006, 38, 795. [CrossRef]

16. Parsons, P.; Barrow, J.D. New inflation from old. Class. Quantum Grav. 1995, 12, 1715. [CrossRef]

17. Barrow, J.D.; Paliathanasis, A. Reconstructions of the dark-energy equation of state and the inflationary potential. Gen. Rel. Gravit. 2018, 50, 82. [CrossRef]

18. Pailas, T.; Dimakis, N.; Paliathanasis, A.; Terzis, P.A.; Christodoulakis, T. The infinite dimensional symmetry groups of the Friedmann equation. Phys. Rev. D 2020, 102, 063524. [CrossRef]

19. Christodoulakis, T.; Karagiorgos, A.; Zampeli, A. Symmetries in classical and quantum treatment of Einstein's cosmological equations and mini-superspace actions. Symmetry 2018, 10, 70. [CrossRef]

20. Tsamparlis, M.; Paliathanasis, A. Symmetries of differential equations in cosmology. Symmetry 2018, 10, 233. [CrossRef]

21. Gionti, G.; Paliathanasis, A. Duality transformation and conformal equivalent scalar-tensor theories. Mod. Phys. Lett. A 2018, 33, 1850093. [CrossRef]

22. Paliathanasis, A.; Tsamparlis, M.; Basilakos, S.; Barrow, J.D. Classical and quantum solutions in Brans-Dicke cosmology with a perfect fluid. Phys. Rev. D 2016, 93, 043528. [CrossRef]

23. Paliathanasis, A.; Capozziello, S. Noether symmetries and duality transformations in cosmology. Mod. Phys. Lett. A 2016, 31, 1650183. [CrossRef]

24. Zampeli, A.; Pailas, T.; Terzis, T.A.; Christodoulakis, T. Conditional symmetries in axisymmetric quantum cosmologies with scalar fields and the fate of the classical singularities. J. Cosmol. Astropart. Phys. 2016, 5, 66. [CrossRef]

25. Christodoulakis, T.; Dimakis, N.; Terzis, P.A. Lie point and variational symmetries in minisuperspace Einstein gravity. J. Phys. A 2014, 47, 095202. [CrossRef]

26. Dimakis, N.; Christodoulakis, T.; Terzis, P.A. FLRW metric cosmology with a perfect fluid by generating integrals of motion. $J$. Geom. Phys. 2012, 77, 97. [CrossRef]

27. Basilakos, S.; Tsamparlis, M.; Paliathanasis, A. Using the Noether symmetry approach to probe the nature of dark energy. Phys. Rev. D 2011, 83, 103512. [CrossRef]

28. Paliathanasis, A.; Tsamparlis, M.; Basilakos, S. Constraints and analytical solutions of $f(R)$ theories of gravity using Noether symmetries. Phys. Rev. D 2011, 84, 123514. [CrossRef]

29. Alberto Vazquez, J.; Hee, S.; Hobson, M.P.; Lasenby, A.N.; Ibison, M.; Bridges, M. Observational constraints on conformal time symmetry, missing matter and double dark energy. J. Cosmol. Astropart. Phys. 2018, 7, 62. [CrossRef]

30. Ben Achour, J.; Livine, E.R. Cosmology as a CFT 1 . J. High Energy Phys. 2019, 12, 31. [CrossRef]

31. Ben Achour, J.; Livine, E.R. The cosmological constant from conformal transformations: Möbius invariance and Schwarzian action. Class. Quantum Grav. 2020, 37, 215001. [CrossRef]

32. Ben Achour, J.; Livine, E.R. Conformal structure of FLRW cosmology: Spinorial representation and the so(3,2) algebra of observables. J. High Energy Phys. 2020, 3, 67. [CrossRef]

33. Dussault, S.; Faraoni, V. A new symmetry of the spatially flat Einstein-Friedmann equations. Eur. Phys. J. C 2020, 80, 1002. [CrossRef]

34. Barrow, J.D. Relativistic cosmology and the regularization of orbits. Observatory 1993, 113, 210. 
35. Rosu, H.C. Darboux class of cosmological fluids with time-dependent adiabatic indices. Mod. Phys. Lett. A 2000, 15, 979. [CrossRef]

36. Nowakowski, M.; Rosu, H.C. Newton's laws of motion in form of Riccati equation. Phys. Rev. E 2002, 65, 047602. [CrossRef]

37. Rosu, H.C.; Ojeda-May, P. Supersymmetry of FRW barotropic cosmologies. Int. J. Theor. Phys. 2006, 45, 1191. [CrossRef]

38. Rosu, H.C.; Ibarra-Junquera, V. FRW barotropic zero modes: Dynamical systems observability. Appl. Math. Sci. 2007, 1, 843. [CrossRef]

39. Rosu, H.C.; Khmelnytskaya, K.V. Shifted Riccati procedure: Application to conformal barotropic FRW cosmologies. SIGMA 2011, 7, 13. [CrossRef]

40. Rosu, H.C.; Khmelnytskaya, K.V. Inhomogeneous barotropic FRW cosmologies in conformal time. Mod. Phys. Lett. A 2013, 28, 1340017. [CrossRef]

41. Rosu, H.C.; Mancas, S.C.; Chen, P. Barotropic FRW cosmologies with Chiellini damping in comoving time. Mod. Phys. Lett. A 2015, 30, 1550100. [CrossRef]

42. Harko, T.; Lobo, F.S.N.; Mak, M.K. A Riccati equation based approach to isotropic scalar field cosmologies. Int. J. Mod. Phys. D 2014, 23, 1450063. [CrossRef]

43. Schuch, D. Quantum Theory from a Nonlinear Perspective: Riccati Equations in Fundamental Physics; Springer: New York, NY, USA, 2018.

44. Cari nena, J.F.; Ra nada, M.F.; Santander, M. Lagrangian Formalism for nonlinear second-order Riccati Systems: One-dimensional Integrability and two-dimensional Superintegrability. J. Math. Phys. 2005, 46, 062703. [CrossRef]

45. Aref'eva, I.Y.; Koshelev, A.S.; Vernov, S.Y. Exact solution in a string cosmological model. Theor. Math. Phys. 2006, 148, 895. [CrossRef]

46. Salopek, D.S.; Bond, J.R. Nonlinear evolution of long-wavelength metric fluctuations in inflationary models. Phys. Rev. D 1990, 42, 3936. [CrossRef]

47. Yurov, A.V.; Yurov, V.A. Friedman versus Abel equations: A connection unraveled. J. Math. Phys. 2010, 51, 082503. [CrossRef]

48. Hawkins, R.M.; Lidsey, J.E. The Ermakov-Pinney equation in scalar field cosmologies. Phys. Rev. D 2002, 66, 023523. [CrossRef]

49. Fomin, I.V.; Chervon, S.V.; Maharaj, S.D. A new look at the Schrödinger equation in exact scalar field cosmology. Int. J. Geom. Meth. Mod. Phys. 2018, 16, 1950022. [CrossRef]

50. Barbosa-Cendejas, N.; Reyes, M.A. The Schrodinger picture of standard cosmology. arXiv 2009, arXiv:1001.0084.

51. Chen, S.; Gibbons, G.W.; Li, Y.; Yang, Y. Friedmann's Equations in All Dimensions and Chebyshev's Theorem. J. Cosmol. Astropart. Phys. 2014, 1412, 35. [CrossRef]

52. Chen, S.; Gibbons, G.W.; Yang, Y. Explicit integration of Friedmann's equation with nonlinear equations of state. J. Cosmol. Astropart. Phys. 2015, 2015, 20. [CrossRef]

53. Chen, S.; Gibbons, G.W.; Yang, Y. Friedmann-Lemaitre cosmologies via roulettes and other analytic methods. J. Cosmol. Astropart. Phys. 2015, 10, 56. [CrossRef]

54. Chebyshev, M.P. L'intégration des différentielles irrationnelles. J. Math. Pures Appl. 1853, 18, 87.

55. Marchisotto, E.A.; Zakeri, G.-A. An invitation to integration in finite terms. College Math. J. 1994, 25, 295. [CrossRef]

56. Faraoni, V. Natural phenomena described by the same equation. Eur. J. Phys. 2020, 41, 054002. [CrossRef] 